\title{
Effect of purchasing and marketing integration on new product development speed: The moderating role of environmental dynamism
}

\author{
González-Zapatero, C. ${ }^{\mathrm{a},{ }^{,},}$, González-Benito, J. ${ }^{\mathrm{a}}$, Lannelongue, G. ${ }^{\mathrm{a}}$ \\ a Universidad de Salamanca, IME Instituto Multidisciplinar de Empresa, Campus Miguel de Unamuno, Salamanca, Spain
}

\begin{abstract}
A B S T R A C T
The increasing relevance of purchasing-marketing functional integration (PMFI) has drawn scholars' attention in recent years. However, more empirical research is still needed that adopts a contingent approach and studies the differentiated role each of these two functions plays in PMFI. Based on Information Processing Theory, the two flows of information that PMFI requires, from marketing to purchasing and vice versa, are used as a PMFI proxy. The study posits a positive impact of these two information flows on a typical NPD performance indicator, namely, its speed, and a positive moderation of environmental dynamism on that effect. Data from 141 Spanish firms are used to conduct a moderated multiple regression analysis to test these effects, showing that the marketing information impact of NPD speed is positive regardless of the level of environmental dynamism. However, the effect of purchasing information on NPD speed is positive when the rate of environmental dynamism is medium or high, but negative when it is low. These results will help managers to assess when each one of these flows should be promoted. Above all, they stress the need to control for possible asymmetries in the role the different functions play in functional integration.
\end{abstract}

\section{ARTICLE INFO}

Keywords:

Product development;

Innovation speed;

Purchasing and marketing

integration;

Environmental dynamism;

Information processing theory

*Corresponding author:

carmengz@usal.es

(González-Zapatero, C.)

Article history:

Received 22 June 2018

Revised 24 April 2019

Accepted 9 May 2019

(C) 2019 CPE, University of Maribor. All rights reserved.

\section{References}

[1] Narver, J.C., Slater, S.F. (1990). The effect of a market orientation on business profitability, Journal of Marketing, Vol. 54, No. 4, 20-35, doi: 10.1177/002224299005400403.

[2] Leenders, M.A.A.M., Wierenga, B. (2002). The effectiveness of different mechanisms for integrating marketing and R\&D, The Journal of Product Innovation Management, Vol. 19, No. 4, 305-307, doi: 10.1111/1540-5885.194 $\underline{0305}$.

[3] Swink, M., Song, M. (2007). Effects of marketing-manufacturing integration on new product development time and competitive advantage, Journal of Operations Management, Vol. 25, No. 1, 203-217, doi: 10.1016/j.jom. 2006.03.001.

[4] Sheth, J.N., Sharma, A., Iyer, G.R. (2009). Why integrating purchasing with marketing is both inevitable and beneficial, Industrial Marketing Management, Vol. 38, No. 8, 865-871, doi: 10.1016/i.indmarman.2008.12.021.

[5] Smirnova, M., Henneberg, S.C., Ashnai, B., Naudé, P., Mouzas, S. (2011). Understanding the role of marketingpurchasing collaboration in industrial markets: The case of Russia, Industrial Marketing Management, Vol. 40, No. 1, 54-64, doi: 10.1016/j.indmarman.2010.09.010.

[6] Wagner, S.M., Eggert, A. (2016). Co-management of purchasing and marketing: Why, when and how?, Industrial Marketing Management, Vol. 52, 27-36, doi: 10.1016/j.indmarman.2015.07.012.

[7] Toon, M.A., Morgan, R.E., Lindgreen, A., Vanhamme, J., Hingley, M.K. (2016). Processes and integration in the interaction of purchasing and marketing: Considering synergy and symbiosis, Industrial Marketing Management, Vol. 52, 74-81, doi: 10.1016/j.indmarman.2015.07.014. 
[8] Ziggers, G.W., Henseler, J. (2016). The reinforcing effect of a firm's customer orientation and supply-base orientation on performance, Industrial Marketing Management, Vol. 52, 18-26, doi: 10.1016/i.indmarman.2015. 07.011.

[9] Matthyssens, P., Bocconcelli, R., Pagano, A., Quintens, L. (2016). Aligning marketing and purchasing for new value creation, Industrial Marketing Management, Vol. 52, 60-73, doi: 10.1016/i.indmarman.2015.07.016.

[10] Luzzini, D., Ronchi, S. (2016). Cinderella purchasing transformation: Linking purchasing status to purchasing practices and business performance, Production Planning \& Control, Vol. 27, No. 10, 787-796, doi: 10.1080/ 09537287.2015 .1137986$.

[11] Nazari-Shirkouhi, S., Keramati, A., Rezaie, K. (2015). Investigating the effects of customer relationship management and supplier relationship management on new product development, Tehnički Vjesnik - Technical Gazette, Vol. 22, No. 1, 191-200, doi: 10.17559/TV-20140623130536.

[12] Swink, M. (2003). Completing projects on-time: How project accelerations affect new product development, Journal of Engineering and Technology Management, Vol. 20, No. 4, 319-344, doi: 10.1016/i.jengtecman.2003. $\underline{08.002}$

[13] Carbonell, P., Rodríguez, A.I. (2006). Designing teams for speedy product development: The moderating effect of technological complexity, Journal of Business Research, Vol. 59, No. 2, 225-232, doi: 10.1016/i.jbusres.2005. $\underline{08.002}$.

[14] Frankel, R., Mollenkopf, D.A. (2015). Cross-functional integration revisited: Exploring the conceptual elephant, Journal of Business Logistics, Vol. 36, No. 1, 18-24, doi: 10.1111/ibl.12081.

[15] Turkulainen, V., Ketokivi, M. (2012). Cross-functional integration and performance: What are the real benefits?, International Journal of Operations \& Production Management, Vol. 32, No. 4, 447-467, doi: 10.1108/01443571 211223095.

[16] Miranda González, F.J., Bañegil Palacios, T.M. (2002). The effect of new product development techniques on new product success in Spanish firms, Industrial Marketing Management, Vol. 31, No. 3, 261-271, doi: 10.1016/ S0019-8501(00)00150-4.

[17] Galbraith, J.R. (1974). Organization design: An information processing view, INFORMS Journal on Applied Analytics, Vol. 4, No. 3, 28-36, doi: 10.1287/inte.4.3.28.

[18] Tushman, M.L., Nadler, D.A. (1978). Information processing as an integrating concept in organizational design, Academy of Management Review, Vol. 3, No. 3, 613-624, doi: 10.5465/amr.1978.4305791.

[19] Pagell, M. (2004). Understanding the factors that enable and inhibit the integration of operations, purchasing and logistics, Journal of Operations Management, Vol. 22, No. 5, 459-487, doi: 10.1016/i.jom.2004.05.008.

[20] Swink, M., Narasimhan, R., Wang, C. (2007). Managing beyond the factory walls: Effects of four types of strategic integration on manufacturing plant performance, Journal of Operations Management, Vol. 25, No. 1, 148-164, doi: 10.1016/i.jom.2006.02.006.

[21] Schoenherr, T., Swink, M. (2012). Revisiting the arcs of integration: Cross-validations and extensions, Journal of Operations Management, Vol. 30, No. 1-2, 99-115, doi: 10.1016/i.jom.2011.09.001.

[22] Swink, M., Schoenherr, T. (2015). The effects of cross-functional integration on profitability, process efficiency, and asset productivity, Journal of Business Logistics, Vol. 36, No. 1, 69-87, doi: 10.1111/jbl.12070.

[23] Hult, G.T.M., Ketchen, D.J., Slater, S.F. (2004). Information processing, knowledge development, and strategic supply chain performance, Academy of Management Journal, Vol. 47, No. 2, 241-253, doi: 10.5465/20159575.

[24] Miller, D., Friesen, P.H. (1983). Strategy-making and environment: The third link, Strategic Management Journal, Vol. 4, No. 3, 221-235, doi: 10.1002/smj.4250040304.

[25] González-Benito, J., Reis da Rocha, D., Queiruga, D. (2010). The environment as a determining factor of purchasing and supply strategy: An empirical analysis of Brazilian firms, International Journal of Production Economics, Vol. 124, No. 1, 1-10, doi: 10.1016/j.ijpe.2009.08.032.

[26] Swink, M., Song, M., (2007). Effects of marketing-manufacturing integration on new product development time and competitive advantage, Journal of Operations Management, Vol. 25, No. 1, 203-217, doi: 10.1016/i.jom. 2006.03.001.

[27] Podsakoff, P.M., Organ, D.W. (1986). Self-reports in organizational research: Problems and prospects, Journal of Management, Vol. 12, No. 4, 531-544, doi: 10.1177/014920638601200408.

[28] Podsakoff, P.M., MacKenzie, S.B., Lee, J.-Y., Podsakoff, N.P. (2003). Common method biases in behavioral research: A critical review of the literature and recommended remedies, Journal of Applied Psychology, Vol. 88, No. 5, 879-903, doi: 10.1037/0021-9010.88.5.879.

[29] Gupta, A.K., Raj, S.P., Wilemon, D. (1986). A model for studying R\&D - Marketing interface in the product innovation process, Journal of Marketing, Vol. 50, No. 2, 7-17, doi: 10.1177/002224298605000201.

[30] Chau, P.Y.K. (1997). Reexamining a model for evaluating information center success using a structural equation modeling approach, Decision Sciences, Vol. 28, No. 2, 309-334, doi: 10.1111/i.1540-5915.1997.tb01313.x.

[31] Hair, J.F., Tatham, R.L., Anderson, R.E., Black, W.C. (1998). Multivariate data analysis, 5th edition, Prentice-Hall International, New York, USA.

[32] Diamantopoulos, A., Winklhofer, H.M. (2001). Index construction with formative indicators: An alternative to scale development, Journal of Marketing Research, Vol. 38, No. 2, 269-277, doi: 10.1509/jmkr.38.2.269.18845.

[33] Jarvis, C.B., MacKenzie, S.B., Podsakoff, P.M. (2003). A critical review of construct indicators and measurement model misspecification in marketing and consumer research, Journal of Consumer Research, Vol. 30, No. 2, 199218, doi: $10.1086 / 376806$.

[34] Kessler, E.H., Bierly, P.E. (2002). Is faster really better? An empirical test of the implications of innovation speed, IEEE Transactions on Engineering Management, Vol. 49, No. 1, 2-12, doi: 10.1109/17.985742. 
[35] Chen, J., Reilly, R.R., Lynn, G.S. (2005). The impacts of speed-to-market on new product success: The moderating effects of uncertainty, IEEE Transactions on Engineering Management, Vol. 52, No. 2, 199-212, doi: 10.1109/ TEM.2005.844926.

[36] Gonzalez-Zapatero, C., Gonzalez-Benito, J., Lannelongue, G. (2016). Antecedents of functional integration during new product development: The purchasing-marketing link, Industrial Marketing Management, Vol. 52, 47-59, doi: 10.1016/j.indmarman.2015.07.015.

[37] Takeuchi, H., Nonaka, I. (1986). The new new product development game, Harvard Business Review, Vol. 64, No. 1, 137-146.

[38] Vorkapić, M., Radovanović, F., Ćoćkalo, D., Đorđević, D. (2017). NPD in small manufacturing enterprises in Serbia, Tehnički Vjesnik - Technical Gazette, No. 24, No. 1, 327-332, doi: 10.17559/TV-20150807185156.

[39] Urban, G.L., Hauser, J.R., (1993). Design and marketing of new products, 2nd edition, Englewood Cliffs, PrenticeHall International, New York, USA.

[40] Yin, F.P., Gao, Q., Ji, X. (2017). Performance modelling based on value analysis for improving product development process architecture, Advances in Production Engineering \& Management, Vol. 12, No. 1, 17-28, doi: 10.14743/apem2017.1.236

[41] Xu, Z., Frankwick, G.L., Ramirez, E. (2016). Effects of big data analytics and traditional marketing analytics on new product success: A knowledge fusion perspective, Journal of Business Research, Vol. 69, No. 5, 1562-1566, doi: 10.1016/i.jbusres.2015.10.017.

[42] Wang, G., Gunasekaran, A., Ngai, E.W.T., Papadopoulos, T. (2016). Big data analytics in logistics and supply chain management: Certain investigations for research and applications, International Journal of Production Economics, Vol. 176, 98-110, doi: 10.1016/j.ijpe.2016.03.014.

[43] Yang, Y., Fu, C., Chen, Y.-W., Xu, D.-L., Yang, S.-L. (2016). A belief rule based expert system for predicting consumer preference in new product development, Knowledge-Based Systems, Vol. 94, 105-113, doi: 10.1016/ j.knosys.2015.11.012. 


\title{
Vpliv nakupne in tržne integracije na hitrost razvoja novih izdelkov: Moderirana vloga okoljske dinamike
}

\author{
González-Zapatero, C. ${ }^{\mathrm{a},{ }^{*}}$, González-Benito, J. ${ }^{\mathrm{a}}$, Lannelongue, G. ${ }^{\mathrm{a}}$
}

${ }^{a}$ Universidad de Salamanca, IME Instituto Multidisciplinar de Empresa, Campus Miguel de Unamuno, Salamanca, Spain

\section{POVZETEK}

Vse večja pomembnost nakupno-tržne funkcionalne integracije (PMFI) je v zadnjih letih pritegnila pozornost znanstvenikov. Kljub temu pa je potrebnih še več empiričnih raziskav, ki na podlagi pogojnega pristopa preučijo različno vlogo vsake od teh dveh funkcij v PMFI. Na podlagi teorije obdelave informacij, se informacijska tokova, ki ju PMFI zahteva, od trženja do nakupa in obratno, uporabljata kot nadomestka za PMFI. Študija ugotavlja pozitiven vpliv teh dveh informacijskih tokov na tipičen kazalnik uspešnosti NPD, in sicer na njegovo hitrost in pozitivno moderiranost dinamike okolja. Za preskušanje teh učinkov z moderirano multiplo regresijsko analizo so uporabljeni podatki 141 španskih podjetij, ki kažejo, da je vpliv tržnih informacij na hitrost NPD pozitiven, ne glede na stopnjo dinamike okolja. Vendar je učinek nakupa informacij na hitrost NPD pozitiven, kadar je stopnja dinamike okolja srednja ali visoka, negativen pa, ko je nizka. Rezultati bodo direktorjem podjetij pomagali oceniti, kdaj je treba spodbujati katerega od teh tokov, predvsem pa poudarjajo potrebo po nadzorovanju možnih asimetrij v vlogah, ki jih zavzamejo različne funkcije pri funkcijski integraciji.

\section{PODATKI O ČLANKU}

Ključne besede:

Razvoj izdelkov;

Hitrost inovacij;

Nakupna in tržna integracija;

Okoljska dinamika;

Teorija obdelave informacij

*Kontaktna oseba:

carmengz@usal.es

(González-Zapatero, C.)

Zgodovina članka:

Prejet 22. junija 2018

Popravljen 24. aprila 2019

Sprejet 9. maja 2019 